\title{
CONTINUOUS RANDOM VARIABLES WITH HADAMARD FRACTIONAL INTEGRAL
}

\author{
KHELLAF OULD MELHA AND VAIJANATH LAXMANRAO CHINCHANE
}

\begin{abstract}
In this paper, we establish some new inequalities of expectation and variance of continuous random variables by using the Hadamard fractional integral operator.
\end{abstract}

\section{Introduction}

Integral inequalities play a major role in the development of integral equations, differential equations, probability theory and other fields of science. In the past several years, many authors have found different results about fractional integral inequalities and application by using Riemann-Liouville and Hadamard fractional integral operators and differential operators, see [5, 7, 8, 15]. In 2001, P. Cerone and S. S. Dargomir [6] obtained the bounds for the variance and expectation of continuous random variable whose the probability density function (p.d.f) is defined on finite interval. In [4], authors have established some integral inequalities for the expectation and variance of a random variable having a probability density function $f:[a, b] \rightarrow \mathbb{R}^{+}$. P. Kumar obtained some new inequalities for the moments and higher order central moments of continuous random variable, see [12]. In 2014, Z. Dahamani has introduced new concepts of fractional random variables (by using Riemann-Liouville fractional integral operator), see [9] in the same paper he has established new integral inequalities for the fractional expectation and fractional variance. In [10] Z. Dahamani established some new results and applications of fractional calculus for continuous random variable, also some corollaries on the paper [9] were corrected. Besides the citations mentioned, one may refer to Baenett et al. [3], P. Kumar [13, 14], Anastassiou [1] and Dahmani [11]. Motivated by the above work, in this paper we establish some new inequalities of expectation and variance of continuous random variables by using the Hadamard fractional integral operator.

Received February 24, 2018, accepted August 21, 2018.

2010 Mathematics Subject Classification. 26D10, 26A33, 60E15..

Key words and phrases. Hadamard fractional integral, integral inequality, random variables.

Corresponding author: Khellaf Ould Melha. 


\section{Preliminaries}

This section is devoted to the basic concepts of Hadamard type fractional calculus:

Definition $1([2,7,8])$. The Hadamard fractional integral of order $\alpha \in \mathbb{R}^{+}$of function $f(x)$, for all $x>1$ is defined as

$$
{ }_{H} D_{1, x}^{-\alpha} f(t)=\frac{1}{\Gamma(\alpha)} \int_{1}^{t} \ln \left(\frac{t}{s}\right)^{\alpha-1} f(s) \frac{d s}{s},
$$

where $\Gamma(\alpha)=\int_{0}^{\infty} e^{-u} u^{\alpha-1} d u$.

We introduce also the following new concepts and definitions:

Definition 2. The fractional expectation function of order $\alpha \geq 0$, for a random variable $X$ with a positive probability density function (p.d.f) $\mathrm{f}$ defined on $[a, b]$, with $a \geq 1$, is defined as

$$
E_{X, \alpha}(t)={ }_{H} D_{1, t}^{-\alpha}[t f(t)]=\frac{1}{\Gamma(\alpha)} \int_{a}^{t}\left(\ln \frac{t}{s}\right)^{\alpha-1} s f(s) \frac{d s}{s} ; \alpha \geq 0, a<t<b .
$$

Definition 3. The fractional expectation function of order $\alpha \geq 0$, for a random variable $X-$ $E(X)$ is defined as

$$
\begin{aligned}
E_{X-E(X), \alpha}(t) & ={ }_{H} D_{1, t}^{-\alpha} t f(t) \\
& =\frac{1}{\Gamma(\alpha)} \int_{a}^{t}\left(\ln \frac{t}{s}\right)^{\alpha-1}(s-E(X)) f(s) \frac{d s}{s} ; \alpha \geq 0, a<t<b,
\end{aligned}
$$

where $f:[a, b] \rightarrow \mathbb{R}^{+}$is the p.d.f. of $X$ with $a \geqslant 1$.

For $t=b$, we introduce the following concept:

Definition 4. The fractional expectation function of order $\alpha \geq 0$, for a random variable $X$ with a positive p.d.f. f defined on $[a, b]$, with $a \geq 1$, is defined as

$$
E_{X, \alpha}(t)={ }_{H} D_{1, t}^{-\alpha}[t f(t)]=\frac{1}{\Gamma(\alpha)} \int_{a}^{b}\left(\ln \frac{t}{s}\right)^{\alpha-1} s f(s) \frac{d s}{s} ; \alpha \geq 0 .
$$

Definition 5. The fractional variance function of order $\alpha \geq 0$, for a random variable $X$ having a p.d.f. $f:[a, b] \rightarrow \mathbb{R}^{+}$with $a \geqslant 1$, is defined as

$$
\begin{aligned}
\sigma_{X, \alpha}^{2} & :={ }_{H} D_{1, t}^{-\alpha}\left[(t-E(X))^{2} f(t)\right] \\
& =\frac{1}{\Gamma(\alpha)} \int_{a}^{t}\left(\ln \frac{t}{s}\right)^{\alpha-1}(s-E(X))^{2} f(s) \frac{d s}{s}, \alpha \geq 0, a<t<b .
\end{aligned}
$$

where $E_{X}(t)=\int_{a}^{b} s f(s) d s$ is the classical expectation of $X$. 
Definition 6. The fractional variance function of order $\alpha \geq 0$, for a random variable $X$ with a p.d.f. $f:[a, b] \rightarrow \mathbb{R}^{+}$is defined as

$$
\sigma_{X, \alpha}^{2}:=\frac{1}{\Gamma(\alpha)} \int_{a}^{b}\left(\ln \frac{b}{s}\right)^{\alpha-1}(s-E(X))^{2} f(s) \frac{d s}{s}, \alpha \geq 0, a \geqslant 1 .
$$

where $E(X)=\int_{a}^{b} s f(s) d s$ is the classical expectation of $X$.

\section{Main results}

In this section, we prove some theorems for the continuous random variable having a p.d.f.

Lemma 7. Let $X$ be a continuous random variable having a p.d.f. $f:[a, b] \rightarrow \mathbb{R}^{+}$. Then we have for all $\alpha \geq 0$ :

$$
\sigma_{X, \alpha}^{2}=E_{X^{2}, \alpha}-2 E(X) E_{X, \alpha}+E(X)^{2}{ }_{H} D_{1, x}^{-\alpha}[f(b)] .
$$

Proof. We have

$$
\sigma_{X, \alpha}^{2}=\frac{1}{\Gamma(\alpha)} \int_{a}^{b}\left(\ln \frac{b}{t}\right)^{\alpha-1}\left[t^{2}+E(X)^{2}-2 t E(X)\right] f(t) \frac{d t}{t}
$$

then

$$
\begin{aligned}
\sigma_{X, \alpha}^{2}= & \frac{1}{\Gamma(\alpha)} \int_{a}^{b}\left(\ln \frac{b}{t}\right)^{\alpha-1} t^{2} f(t) \frac{d t}{t}-\frac{2 E(X)}{\Gamma(\alpha)} \int_{a}^{b}\left(\ln \frac{b}{t}\right)^{\alpha-1} t f(t) \frac{d t}{t} \\
& +\frac{E(X)^{2}}{\Gamma(\alpha)} \int_{a}^{b}\left(\ln \frac{b}{t}\right)^{\alpha-1} f(t) \frac{d t}{t}
\end{aligned}
$$

and we obtain

$$
\sigma_{X, \alpha}^{2}=E_{X^{2}, \alpha}-2 E(X) E_{X, \alpha}+E(X)^{2}{ }_{H} D_{1, x}^{-\alpha}[f(b)] .
$$

Theorem 8. Let $X$ be a continuous random variable having a p.d.f. $f:[a, b] \rightarrow \mathbb{R}^{+}$. Then we have

1. For all $\alpha \geq 0, a<t \leq b$,

$$
{ }_{H} D_{1, x}^{-\alpha}[f(t)] \sigma_{X, \alpha}^{2}-\left(E_{X-E(X), \alpha}(t)\right)^{2} \leq\|f\|_{\infty}^{2}\left[2 \frac{\left(\ln \frac{t}{a}\right)^{\alpha}}{\Gamma(\alpha+1)}{ }_{H} D_{1, t}^{-\alpha}\left[t^{2}\right]-2\left({ }_{H} D_{1, t}^{-\alpha}[t]\right)^{2}\right],
$$

provided that $f \in L_{\infty}([a, b])$. 
2. The inequality

$$
{ }_{H} D_{1, t}^{-\alpha}[f(t)] \sigma_{X, \alpha}^{2}-\left(E_{X-E(X), \alpha}(t)\right)^{2} \leq \frac{1}{2}(t-a)^{2}\left({ }_{H} D_{1, t}^{-\alpha}[f(t)]\right),
$$

holds For all $\alpha \geq 0, a<t \leq b$.

Proof. Suppose that for $s, y \in(a, t)$ and $a<t \leq b$

$$
H(t, y)=(g(s)-g(y))(h(s)-h(y))=g(s) h(s)-g(s) h(y)-g(y) h(t)+g(y) h(y) .
$$

Let $f:[a, b] \rightarrow \mathbb{R}^{+}$, multiplying (3) by $\frac{\left(\ln \frac{t}{s}\right)^{\alpha-1}}{\Gamma(\alpha)} p(s)$

$$
\begin{aligned}
\frac{1}{\Gamma(\alpha)} \int_{a}^{t}\left(\ln \frac{t}{s}\right)^{\alpha-1} p(s) H(s, y) \frac{d s}{s}= & { }_{H} D_{1, t}^{-\alpha}[p g h(t)]-h(y)_{H} D_{1, t}^{-\alpha}[p g(t)] \\
& -g(y)_{H} D_{1, t}^{-\alpha}[p h(t)]+g(y) h(y)_{H} D_{1, t}^{-\alpha}[p(t)] .
\end{aligned}
$$

Multiplying (4) by $\frac{\left(\ln \frac{t}{y}\right)^{\alpha-1}}{\Gamma(\alpha)} p(y), y \in(a, t)$ and integrating the resulting identity with respect to $y$ over $(a, t)$, we can write

$$
\begin{aligned}
& \frac{1}{\Gamma^{2}(\alpha)} \int_{a}^{t} \int_{a}^{t}\left(\ln \frac{t}{s}\right)^{\alpha-1}\left(\ln \frac{t}{y}\right)^{\alpha-1} p(y) p(s) H(s, y) \frac{d s}{s} \frac{d y}{y} \\
& =2_{H} D_{1, t}^{-\alpha}[p(t)]{ }_{H} D_{1, t}^{-\alpha}[\operatorname{pgh}(t)]-2_{H} D_{1, t}^{-\alpha}[p h(t)]_{H} D_{1, t}^{-\alpha}[p g(t)] .
\end{aligned}
$$

In (5), taking $p(t)=f(t), g(t)=h(t)=t-E(X), t \in(a, b)$, we have

$$
\begin{aligned}
& \frac{1}{\Gamma^{2}(\alpha)} \int_{a}^{t} \int_{a}^{t}\left(\ln \frac{t}{s}\right)^{\alpha-1}\left(\ln \frac{t}{y}\right)^{\alpha-1} f(y) f(s)(s-y)^{2} \frac{d s}{s} \frac{d y}{y} \\
& =2_{H} D_{1, t}^{-\alpha}[f(t)]{ }_{H} D_{1, t}^{-\alpha}\left[f(t)\left(t-E(X)^{2}\right)\right]-2_{H} D_{1, t}^{-\alpha}[f(t)(t-E(X))]^{2} .
\end{aligned}
$$

On other hand, we have

$$
\begin{aligned}
& \frac{1}{\Gamma^{2}(\alpha)} \int_{a}^{t} \int_{a}^{t}\left(\ln \frac{t}{s}\right)^{\alpha-1}\left(\ln \frac{t}{y}\right)^{\alpha-1} f(y) f(s)(s-y)^{2} \frac{d s}{s} \frac{d y}{y} \\
& \leq\|f\|_{\infty}^{2} \frac{1}{\Gamma^{2}(\alpha)} \int_{a}^{t} \int_{a}^{t}\left(\ln \frac{t}{s}\right)^{\alpha-1}\left(\ln \frac{t}{y}\right)^{\alpha-1}(s-y)^{2} \frac{d s}{s} \frac{d y}{y} \\
& \leq\|f\|_{\infty}^{2}\left[2 \frac{\left(\ln \frac{t}{a}\right)^{\alpha}}{\Gamma(\alpha+1)}{ }_{H} D_{1, t}^{-\alpha}\left[t^{2}\right]-2\left({ }_{H} D_{1, t}^{-\alpha}[t]\right)^{2}\right]
\end{aligned}
$$

Thanks to (6) and (7), we obtain the part (1) of Theorem 8.

For the part (2), we have

$$
\frac{1}{\Gamma^{2}(\alpha)} \int_{a}^{t} \int_{a}^{t}\left(\ln \frac{t}{s}\right)^{\alpha-1}\left(\ln \frac{t}{s}\right)^{\alpha-1} f(y) f(s)(s-y)^{2} \frac{d s}{s} \frac{d y}{y}
$$




$$
\leq \sup _{s, y \in[a, t]}|(s-y)|^{2}\left({ }_{H} D_{1, t}^{-\alpha}[f(t)]\right)^{2}=(t-a)^{2}\left({ }_{H} D_{1, t}^{-\alpha}[f(t)]\right)^{2} .
$$

So by (6) and (8), we obtain the inequality (2).

We shall further generalize Theorem 8 by considering two fractional positive parameters:

Theorem 9. Let $X$ be a continuous random variable having a p.d.f. $f:[a, b] \rightarrow \mathbb{R}^{+}$. Then we have

(a) For all $a<t \leq b, \alpha \geq 0, \beta \geq 0$,

$$
\begin{aligned}
& { }_{H} D_{1, t}^{-\alpha}[f(t)] \sigma_{X, \beta}^{2}+{ }_{H} D_{1, t}^{-\beta}[f(t)] \sigma_{X, \alpha}^{2}-\left(E_{X-E(X), \alpha}(t)\right)\left(E_{X-E(X), \beta}(t)\right) \\
& \quad \leq\|f\|_{\infty}^{2}\left[\frac{\left(\ln \frac{t}{a}\right)^{\alpha}}{\Gamma(\alpha+1)} H_{H} D_{1, t}^{-\beta}\left[t^{2}\right]+\frac{\left(\ln \frac{t}{a}\right)^{\beta}}{\Gamma(\alpha+1)}{ }_{H} D_{1, t}^{-\alpha}\left[t^{2}\right]-\left({ }_{H} D_{1, t}^{-\alpha}[t]\right)\left({ }_{H} D_{1, t}^{-\beta}[t]\right)\right],
\end{aligned}
$$

where $f \in L_{\infty}([a, b])$.

(b) The inequality

$$
\begin{aligned}
& { }_{H} D_{1, t}^{-\alpha}[f(t)] \sigma_{X, \beta}^{2}+{ }_{H} D_{1, t}^{-\beta}[f(t)] \sigma_{X, \alpha}^{2}-\left(E_{X-E(X), \alpha}(t)\right)\left(E_{X-E(X), \beta}(t)\right) \\
& \quad \leq(t-a)^{2}\left({ }_{H} D_{1, t}^{-\alpha}[f(t)]\right)\left({ }_{H} D_{1, t}^{-\beta}[f(t)]\right)
\end{aligned}
$$

holds for any $a<t \leq b, \alpha \geq 0, \beta \geq 0$.

Proof. Multiplying (4) by $\frac{\left(\ln \frac{t}{y}\right)^{\beta-1}}{\Gamma(\beta)} p(y)$, we obtain

$$
\begin{aligned}
& \frac{1}{\Gamma(\alpha) \Gamma(\beta)} \int_{a}^{t} \int_{a}^{t}\left(\ln \frac{t}{s}\right)^{\alpha-1}\left(\ln \frac{t}{y}\right)^{\beta-1} p(y) p(s) H(s, y) \frac{d s}{s} \frac{d y}{y} \\
& ={ }_{H} D_{1, t}^{-\alpha}[p(t)]{ }_{H} D_{1, t}^{-\beta}[p(t) g(t) h(t)]+{ }_{H} D_{1, t}^{-\beta}[p(t)]{ }_{H} D_{1, t}^{-\alpha}[p(t) g(t) h(t)] \\
& \quad-{ }_{H} D_{1, t}^{-\beta}[p(t) h(t)]{ }_{H} D_{1, t}^{-\alpha}[p(t) g(t)]{ }_{H} D_{1, t}^{-\alpha}[p(t) h(t)]_{H} D_{1, t}^{-\beta}[p(t) g(t)],
\end{aligned}
$$

taking $p(t)=f(t), g(t)=h(t)=t-E(X), t \in(a, b)$ in (11), we get:

$$
\begin{aligned}
& \frac{1}{\Gamma(\alpha) \Gamma(\beta)} \int_{a}^{t} \int_{a}^{t}\left(\ln \frac{t}{s}\right)^{\alpha-1}\left(\ln \frac{t}{y}\right)^{\beta-1} f(y) f(s)(s-y)^{2} \frac{d s}{s} \frac{d y}{y} \\
& ={ }_{H} D_{1, t}^{-\alpha}[f(t)]{ }_{H} D_{1, t}^{-\beta}\left[f(t)\left(t-E(X)^{2}\right)\right]{ }_{H} D_{1, t}^{-\beta}[f(t)]{ }_{H} D_{1, t}^{-\alpha}\left[f(t)\left(t-E(X)^{2}\right)\right] \\
& \quad-2_{H} D_{1, t}^{-\alpha}[f(t)(t-E(X))]{ }_{H} D_{1, t}^{-\beta}[f(t)(t-E(X))] .
\end{aligned}
$$

We have also

$$
\frac{1}{\Gamma(\alpha) \Gamma(\beta)} \int_{a}^{t} \int_{a}^{t}\left(\ln \frac{t}{s}\right)^{\alpha-1}\left(\ln \frac{t}{y}\right)^{\beta-1} f(y) f(s)(s-y)^{2} \frac{d s}{s} \frac{d y}{y}
$$




$$
\begin{aligned}
& \leq\|f\|_{\infty}^{2} \frac{1}{\Gamma(\alpha) \Gamma(\beta)} \int_{a}^{t} \int_{a}^{t}\left(\ln \frac{t}{s}\right)^{\alpha-1}\left(\ln \frac{t}{y}\right)^{\beta-1}(s-y)^{2} \frac{d s}{s} \frac{d y}{y} \\
& \leq\|f\|_{\infty}^{2}\left[\frac{\left(\ln \frac{t}{a}\right)^{\alpha}}{\Gamma(\alpha+1)}{ }_{H} D_{1, t}^{-\alpha}\left[t^{2}\right]+\frac{\left(\ln \frac{t}{a}\right)^{\beta}}{\Gamma(\beta+1)} H_{1, t}^{\beta} D_{1}^{-\beta}\left[t^{2}\right]-2\left({ }_{H} D_{1, t}^{-\alpha}[t]\right)\left({ }_{H} D_{1, t}^{-\beta}[t]\right)\right] .
\end{aligned}
$$

Thanks to (12) and (13), we obtain (9).

For the part (10), we us the fact that $\sup _{t, y \in[a, x]}|(s-y)|^{2}=(t-a)^{2}$

$$
\begin{aligned}
& \frac{1}{\Gamma(\alpha) \Gamma(\beta)} \int_{a}^{t} \int_{a}^{t}\left(\ln \frac{t}{s}\right)^{\alpha-1}\left(\ln \frac{t}{y}\right)^{\beta-1} f(y) f(s)(s-y)^{2} \frac{d s}{s} \frac{d y}{y} \\
& \quad \leq(t-a)^{2}\left({ }_{H} D_{1, t}^{-\alpha}[f(t)]\right)\left({ }_{H} D_{1, t}^{-\beta}[f(t)]\right) .
\end{aligned}
$$

And, by (13) and (15), we obtain (10).

Remark 10. In Theorem 9, for $\alpha=\beta$, we obtain Theorem 8 .

\section{References}

[1] G. A. Anastassiou, M. R. Hooshmandal, A. Ghasemi and F. Moftakharzadeh, Monotgomery identities for fractional integral and realated fractional inequalities, J. Inequal. Pure Appl. Math., 10(2009), No.4, 1-6.

[2] D. Baleanu, J. A. T. Machado and C.J. Luo, Fractional Dynamic and Control, Springer, 2012, 159-171.

[3] N. S. Barnett, Some inequalities for random variable whose probability density functions are bounded usung a pre-Gr $\tilde{A} \frac{1}{4}$ ss-type inequality, KYUNGPOOK, Math. J., 40(2000), 299-311.

[4] N. S. Barnett P. Cerone, S. S. Dargomir and J. Roumeliotis, Some inequalities for the expectation and variance of a random variable whose PDF is n-time differentiable, J. Inequal. Pure. Appl. Math., 1 (2000), 1-29.

[5] S. Belarbi and Z. Dahmani, On some new fractional integral inequalities, J. Inequal. Pure. Appl. Math., 10 (3) (2009), 1-12.

[6] P. Cerone and S. S. Dargomir, On some inequalities for the expectation and variance, Korean J. Comput. Appl. Math., 8 (2001), 357-380.

[7] V. L. Chinchane and D. B. Pachpatte, New fractional inequalities viz Hadamard fractionalintegral, Internat. J. Functional. Analyisis, Operator. Theory Math., 8 (2001), 357-380.

[8] V. L. Chinchane and D. B. Pachpatte, On some new GrÃss-type inequality using Hadamar fractional integral operator, Journal of Fractional Calculusand Application, 5 (3S) (2014), 1-10.

[9] Z. Dahmani, Fractional integral inequalities for continous randon variables, Malaya Journal of Matematik, 2 (2) (2014), 172-179.

[10] Z. Dahmani, New applications of fractional calculus on probabilistic random variables, Acta Math. Univ. Comenianae, LXXXVI 2(2017), 299-307.

[11] Z. Dahmani, A. E. Bouziane, M. Houas and M. Z. Sarikaya, New W-weighted concepts for continuous random variables with application, Note di Matematica, Note Mat., 37(2017), No.1, 23-40.

[12] P. Kumar, Moment inequalities of a random variable defined over a finit interval, J. Inequal. Pure Appl. Math., 3 (3) (2002), 1-624.

[13] P. Kumar, Inequalities involving moments of a continuous random variable over a finit interval, Computers and Mathematics with Application, 48 (2004), 257-273.

[14] P. Kumar, The Ostrowski type moments integral inequalities and moment-bounds for continuous random variable, Comput. Maths. Appl., 49 (2005), 1929-1940. 
[15] W. Sudsutad, S. K. Ntouyas and J. Tariboon, Fractional integral inequalitis via Hadamard's fractional integral, Abstract and Applied Analysis, 2014 (2014), Article ID 563096, 11 page.

Department of Mathematics, UHB, University of Chlef, Algeria.

E-mail: k.ouldmelha@univ-chlef.dz

Department of Mathematics, Deogiri Institute of Engineering and Management, Studies Aurangabad-431005, INDIA.

E-mail: chinchane85@gmail.com 